\title{
Fatigue Life Prediction and Structural Optimization on Torsion Shaft of Tracked Vehicle
}

\author{
Sihong Yan ${ }^{1, a}$, Fenghe $\mathrm{Tao}^{1}$,Changzhi $\mathrm{Jia}^{1}$ and Guangsheng Liu ${ }^{1}$ \\ ${ }^{1}$ Department One, Mechanical Engineering College, Shi Jia Zhuang, 050003,China \\ aysh5100@163.com
}

\begin{abstract}
Keywords: Torsion shaft, ANSYS, Durability, Optimization, Parameterization
Abstract. In order to make a reasonable use of durability and design life, the mechanical components are optimized in many aspects. From the practical point of view, the parametric modelling of the torsion shaft was carried out with Solidworks, and the model was transferred to the ANSYS Workbench through the data interface. A parameterized model of torsion shaft was developed based on Solidworks and imported into ANSYS Workbench by data interface. At the same time, based on the ANSYS Workbench, the transient stress analysis and fatigue analysis of the parameterized model are carried out. On this basis, the influence of different structures on the fatigue life of the torsion shaft is analysed, and the structure of the torsion shaft is improved. The optimization of structure of tracked vehicle components is explored, and the study results can widely extended to other engineering field.
\end{abstract}

\section{Introduction}

Torsion shaft is the key component in suspension device of tracked vehicle, its role is to absorb the shock energy when the tracked vehicle traveling. Compared with other elastic components, the torsion shaft is simple in structure, light in weight and long in service life. Torsion shaft is still widely used in engineering ${ }^{[1]}$.

\section{Building the model of torsion shaft}

The three-dimensional model of torsion shaft was established according to drawing in SolidWorks, and define the parameters which are needed for optimization. In order to ensure the mesh model smoothly, prevent the grid size and shape having large differences, guarantee the quality and accuracy of classification unit, simplify the local features, such as the positioning hole and the chamfer, which have tiny impact on strength and rigidity of torsion shaft.

The primary structural parameters of torsion shaft:

$D S \_1=0.08 \mathrm{~m} ; D S \_2=0.06 \mathrm{~m} ; D S \_3=0.066 \mathrm{~m} ;$ Diameter of shaft: $D S \_4=0.05 \mathrm{~m}$; Chamfering: $D S \_5=0.015 \mathrm{~m} ; D S \_6=D S \_7=0.2 \mathrm{~m}$.

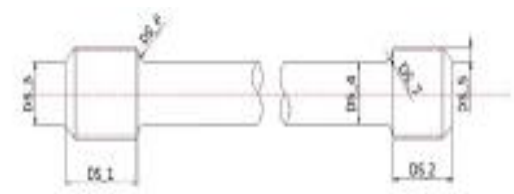

Figure 1. Structural parameters of the torsion shaft.

\section{Finite Element Model Establishment and Stress Analysis}

Finite element model building is making full use of the SolidWorks and ANSYS Workbench software fast-transmission advantage, torsion shaft model is imported into DM (Design Modeler) environment in SolidWorks, and adding material properties and meshing on three-dimensional model which is imported. Stress analysis is that adding constraint boundary conditions and loading on the finite element model, and to find the solution of stress and strain. 


\section{The establishment of finite element model of torsion shaft}

After the three-dimensional model import in ANSYS Workbench, need to add the corresponding materials on model. Check the related information, the material of torsion shaft is $45 \mathrm{CrNiMoVA}$, sample of the specifications for heat treatment as follows ${ }^{[2]}: 870 \pm 10^{\circ} \mathrm{C}$ oil quenching, $430 \pm 20^{\circ} \mathrm{C}$ tempering and oil cooling. Conventional mechanical properties after heat treatment as follows: tensile strength $\sigma_{b}=1.553 \times 10^{9} \mathrm{~Pa}$, tensile yield strength $\sigma_{a}=1.347 \times 10^{9} \mathrm{~Pa}$, Shear yield strength $\tau_{b} \approx 0.8 \sigma_{b}=1.242 \times 10^{9} \mathrm{~Pa}$, Elastic modulus $E=2.06 \times 10^{11} \mathrm{~Pa}$, Poisson ratio $\mu=0.3$, Density $\rho=7830 \mathrm{~kg} / \mathrm{m}^{3}$.

\section{Meshing}

ANSYS Workbench brings meshing tool platform and has a variety of different meshing method for three-dimensional geometry. Automatic mesh method is automatic switching between tetrahedron partition and scanning type partition, generating tetrahedron and hexahedron units in the model.

The three-dimensional model was meshed by the method of automatic mesh grid in this paper, and local unit size is $6 \mathrm{~mm}$. The unit number is 50540, node number is 222833 .

\section{Boundary conditions and loads}

When tracked vehicles driving on the road, the wheel run into obstacles and the torsion shaft turn around, then using its torsional deformation to play a role of elasticity suspension. For convenience of analysis, this progress is equated to the short spline of torsion shaft is fixed and the long spline of torsion shaft is turned.

$$
\begin{aligned}
& \theta_{j}=\frac{M_{j} \times L}{G \times J_{p}}=\frac{P_{j} \times a \times L \cos \alpha_{j}}{G \times J_{p}}=22.89 \\
& \theta_{\text {max }}=\theta_{j} \cdot \frac{\tau_{\text {max }}}{\tau_{j}}=59.77^{\circ} \\
& M_{\text {max }}=\frac{G J_{p} \theta_{\text {max }}}{L}=2.686 \times 10^{4} \mathrm{~N} \cdot \mathrm{m}
\end{aligned}
$$

In the formula: $M_{\max }$ is maximum torque of torsion shaft; $G$ is shear elastic modulus of torsion shaft; $\theta_{\max }$ is maximum torsional angle of torsion shaft; $J_{p}$ is sectional inertia moment of torsion shaft; $L$ is work length of torsion shaft; $P_{j}$ is static load; $a$ is length of road wheel arm; $\theta_{j}$ is static torsional angle; $\tau_{\max }$ is maximum shear stress; $\tau_{j}$ is shear stress.

The above result is maximum torque load of torsion shaft under the static strength design of torsion shaft. But in the process of tracked vehicle real driving, torsion bearing torque by the random load, and generally far less than the maximum torque load.

In this study, based on the virtual prototype model of tracked vehicle, the virtual travel simulation was carried out on the commonly used digital road surfaces of tracked vehicle, and the random alternating torque dynamic load spectrum of the torsion shaft was obtained, the maximum torque load of torsion shaft is $12972 \mathrm{~N} \cdot \mathrm{m}$.

Maximum torque load of test results was applied on the torsion shaft torque loading end, and fixed constraint was added on the fixed end of torsion shaft. By the calculation, maximum shear stress of torsion shaft is $5.57 \times 10^{8} \mathrm{~Pa}$, as shown in the figure 2 . We can learn from the figure 2 , the point of maximum shear stress of torsion shaft is on the journal which between rod and spline. 


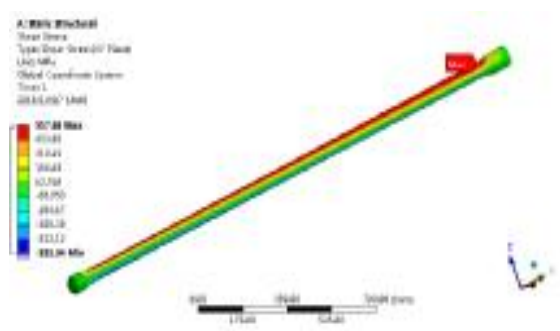

Figure 2. Shear stress diagram.

\section{Analysis of durability fatigue of torsion}

Fatigue is an important reason of structure failure. High cycle fatigue and low cycle fatigue are the mainly types of fatigue failure. The characteristics of high cycle fatigue: under stress of parts or components is low and usually less than the yield limit. Before accident happened, this kind of fatigue failure is not easy to find, but will cause great damage to the components after the occurrence, and commonly exists in mechanical components. The main load of torsion shaft of tracked vehicle is high cycle fatigue load, on this basis, fatigue life of torsion shaft is analysed through nominal stress method in this study.

\section{The S-N curve of the material}

For fatigue life prediction of torsion shaft under random load, the fatigue performance data of torsion shaft material are needed, which are S-N curve data. S-N curve is expressed as:

$$
\log N=a+b \log S
$$

In the formula: $S$ is the stress; $N$ is the number of cycles.

Normally, the S-N curve refers to a fatigue curve with a failure probability of $50 \%$ in the works. When the failure probability is $50 \%$, the material constant $a=32.3665$, the material constant $b=-$ 9.5907. And the $\mathrm{S}-\mathrm{N}$ curve of the $45 \mathrm{CrNiMoVA}$ is drawn according to the related literature ${ }^{[3]}$.

\section{Correction formula of material}

When using the S-N curve of the material to calculate the durability fatigue of the parts, we need to consider the influence of the average stress and introduce the material correction formula. Goodman formula of ANSYS Workbench is suitable for high strength steel, and the correction results more accord to the actual situation, so we choose Goodman formula to amend in this paper. Goodman formula is ${ }^{[4]}$ :

$$
\sigma_{a}^{\prime}=\sigma_{-1}\left(1+\frac{\sigma_{m}}{\sigma_{b}^{\prime}}\right)
$$

In the formula: $\sigma_{a}^{\prime}$ is stress amplitude of asymmetric circulation; $\sigma_{-1}$ is Symmetrical cyclic fatigue limit; ${ }^{\sigma_{m}}$ is the stress of asymmetric circulation; $\sigma_{b}^{\prime}$ is the limit of static strength.

\section{Fatigue durability simulation of torsion shaft}

The dynamic load is added to the finite element model in the way of load step. The time interval and load amplitude are the same as the measured data. The average stress correction theory is Goodman and the analysis type is Stress life ${ }^{[5]}$. Based on the analysis of the transient stress of the torsion shaft in the second part, the fatigue life of the torsion shaft is predicted and simulated. The result of simulation is shown in figure 3 . 


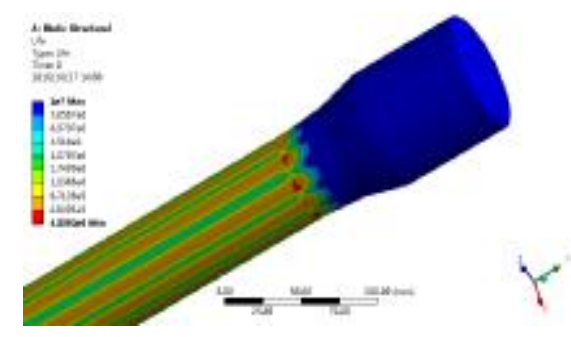

Figure 3. Durability fatigue diagram of torsion shaft.

The results of the fatigue life prediction of the torsion shaft under the dynamic load of torque in figure 3 show that the stress cycle can support more than $1.2 \times 10^{6}$ times in most parts of the torsion shaft. The minimum stress cycle occurs at the site is the journal, the number of cycles is $4.3 \times 10^{5}$ times, and life mileage is $7.029 \times 10^{7}$ meters. The comparison between the predicted location of the minimum durability fatigue and the fracture location of the torsion shaft during the actual test of the tracked vehicle has a good consistency. This shows that the simulation method is correct and the simulation results of torsion shaft are accurate. The actual life mileage that is far more than the limit of overhaul period of the torque shaft is greater than $7.029 \times 10^{7}$ meters.

Through the analysis of the results of transient stress and fatigue life analysis and measurement of the self-weight of the torsion axis is $36.4 \mathrm{~kg}$. The conclusion is drawn that the torsion shaft satisfies the condition of stress and fatigue life, and the quality is heavier. It is shown that there is some optimization space in the torsion shaft, which can be used to optimize the structure.

\section{Research on structure improvement of torsion shaft}

The traditional design method of the torsion shaft is the safety factor method for the maximum load, and the design of the torsion shaft is bulky. According to the results of the simulation analysis, it can be seen that the durability life of the torsion shaft is much longer than the design life. In some equipment-specific maintenance guidelines, the component must be replaced when it reaches its design life ${ }^{[6]}$. Therefore, this paper studies the structure optimization of the torsion shaft, reduces the quality of the torsion shaft under the premise of satisfying the design requirements of the torsion shaft.

Refer to the mechanical design manual and other related design data, the initial diameter of the shaft is set to $35 \%$ of the outer diameter of the torsion shaft. The three-dimensional solid model of the torsion shaft with different inner diameter is established, and its fatigue life is analyzed and predicted by simulation method.

The fatigue life of the hollow torsion shaft is lower than that of the solid torsional shaft, but the fatigue lie is still within the design requirements and the mass structure is reduced. So the hollow torsion shaft instead of solid torsion shaft is feasible. Under the condition of satisfying the design fatigue life of $10^{7} \mathrm{~m}$, the best inner diameter of the torsion shaft is $23.7 \mathrm{~mm}$ which is calculated by interpolation method. And the mass of the torsion shaft is $30.2 \mathrm{~kg}$, reduced by nearly $17 \%$.

\section{Conclusion}

Torsion shaft is still the most used suspension structure of tracked vehicle. The requirement for high reliability and high makes the study of the accurate prediction of the fatigue life and the new structure of the mechanical components becomes a hot topic in today. In this study, the fatigue life analysis method of torsion shaft based on ANSYS Workbench is presented. The modelling and simulation of the torsion shaft are completed, and the results are compared with the actual situation, indicating the applicability of the method. 
1) The dynamic stress and durability fatigue life of the torsion shaft of the tracked vehicle are analyzed under the dynamic load obtained from the virtual prototype simulation test. The accuracy of the model and the usability of the method are verified by comparing with the actual fracture site and service life.

2) Based on the fatigue life prediction method of torsion shaft, the influence of different inner diameter values on fatigue life and structure quality of hollow torsion shafts was studied. And based on the influence rule, the inner diameter of the torsion shaft under the condition of the lightest weight and enough fatigue life is calculated. In this study, the optimization of the structure of the torsion shaft is explored and research methods have a certain guiding significance.

\section{Acknowledgements}

This research was supported by the Science and Technology Project of Hebei Province(No.15210701D). All support is gratefully acknowledged. And I would like to express my gratitude to all those who have helped me during the writing of this thesis. I gratefully acknowledge the help of my supervisor ProfessorTao Fenghe.

\section{References}

[1] Q. Liu, Y.P. Qian, G.Z. Ji, et al. Durability-based design optimization of torsion shaft under ramdom loading, Acta Armam., 36, 5 (2015)

[2] F. Gao, C. Hai, Z.H. Ye, et al. Research and production of steel 45CrNiMoVA for light automobile torsion rod spring, Special Steel., 22, 5 (2001)

[3] G.F. Zhou, X.Y. Li, Y.W. Shi, et al. Numerical simulation of the working process and life prediction of a tank torsion shaft, Journal of Mechanical Strength., 28, 4 (2006)

[4] G.F. Su, Y.C. Bo, J.J. Kong, et al. Fatigue life analysis and research on rod end spherical bearings under dynamic stress, J. Machine Design., 30, 3 (2013)

[5] W. Sun, B. Ma, J. MA, et al. Reliability analysis of suspension device in the refit of a tracked vehicle, Noise Vibra Contrl., 34, 4 (2014)

[6] Z. Liu, F.H. Tao, C.Z. Jia. Fatigue life prediction on drive shaft of tracked vehicle and structure optimization, J. Eng Desing., 22, 5 (2015) 